\title{
Professionelles Handeln in der Sozialen Arbeit
}

\begin{abstract}
Sicht der Praktiker_innen
In der Disziplin Soziale Arbeit ist häufig die Rede von der Bedeutung professionellen Handelns. Aber was verstehen Praktiker_innen eigentlich darunter? Spiegelt ihr Verständnis von professionellem Handeln die vielfachen Erwartungen, die an die Profession herangetragen werden?
\end{abstract}

$\mathrm{V}$ on professionellem Handeln spricht man, wenn sich gekonnte Fachlichkeit in qualitativ hochwertiger Arbeit ausdrückt (Ehlert 2019, o. S.). Gemeint ist in der Sozialen Arbeit ein Handeln, das nicht schematisch vorgeht und sich nicht darin erschöpft, „Handlungsanleitungen“ zu befolgen, da auf jeden Fall individuell eingegangen werden muss, weil er sich jeweils anders darstellt (z. B. Pantucek-Eisenbacher 2015). Prägnant formuliert: „Professionelles Handeln ist im Kern immer dann gefordert, wenn komplexe Problemlagen vorliegen, für die es keine einfachen und eindeutigen Lösungen gibt sowie wenn für das Verständnis der Problemlagen mehr als Alltagswissen und für mögliche Formen des Umgangs mit diesen spezialisiertes Fachwissen und Methodenkenntnisse erforderlich sind." (Scherr 2018, S. 9).

\begin{tabular}{|l|l|} 
Maria Ohling \\
Hochschule für angewandte Wissenschaften Landshut, \\
Landshut, Deutschland \\
Dr. phil., Dipl. Pädagogin, Dipl. Sozialpädagogin/Sozialarbeiterin \\
und Systemische Familientherapeutin. Seit 2004 Professorin \\
für Handlungs- und Methodenlehre der Sozialen Arbeit an \\
der Hochschule für angewandte Wissenschaften in Landshut. \\
Langjährige Tätigkeit als Klinische Sozialarbeiterin in \\
unterschiedlichen Einrichtungen. \\
maria.ohling@haw-landshut.de
\end{tabular}

Zusammenfassung Mit 16 Sozialarbeiter_innen wurden qualitative Interviews geführt zur Frage, was sie unter professionellem Handeln verstehen. Im Vordergrund stehen für die Befragten Methodenwissen und Qualitätsentwicklung. Theoretische und wissenschaftliche Grundlagen sowie Professionsethik kommen weniger in den Sinn. Die Ergebnisse werden in den Kontext anderer empirischer Befunde und in den Diskurs der Sozialen Arbeit gestellt.

Schlüsselwörter Soziale Arbeit, Professionelles Handeln, Methodenwissen - Qualitätsentwicklung, Theoretische und wissenschaftliche Grundlagen, Professionsethik
Ob Sozialarbeiter_innen diesem hohen Anspruch gerecht werden können, wird in der Professionsforschung unterschiedlich diskutiert. Einen guten Überblick über den momentanen Forschungsstand gibt Unterkofler (2018). Demnach bestehen Zweifel daran, ob Sozialarbeiter_innen einen professionellen Habitus ausbilden, wenn es beispielsweise um die Klärung von Auftrag und Zuständigkeit, Diagnostik oder Methodeneinsatz geht oder um die Aneignung theoretischen und wissenschaftlichen Wissens. Allerdings gibt es auch empirische Untersuchungen wie die von Heiner (2004), in der die Studienteilnehmer_innen mehrheitlich als professionell Handelnde eingestuft werden. Unterkofler (2018) schlussfolgert aus ihrer Analyse, dass weitere Forschung auf diesem Gebiet erforderlich ist.

Hier weiteres Licht ins Dunkel zu bringen, ist Anliegen einer Untersuchung, die im Jahre 2017 von der Autorin durchgeführt wurde. Es wird der Frage nachgegangen, was Praktiker_innen selbst unter professionellem Handeln verstehen.

\section{Methode}

Es handelt sich um eine qualitativ angelegte explorative, das heißt nicht repräsentative Studie, die im Rahmen eines Lehrforschungsseminars an der Fakultät Soziale Arbeit der Hochschule Landshut im Jahre 2017 konzipiert und realisiert wurde. In die Untersuchung einbezogen waren 16 Sozialarbeiter_innen $(\mathrm{w}=11, \mathrm{~m}=5)$ aus verschiedenen Berufsfeldern (Kinder- und Jugendhilfe, Wohnungslosenhilfe, Beratungsstellen). Das durchschnittliche Alter der Teilnehmer_ innen beträgt 41 Jahre (Spanne von 23 bis 58 Jahre). Die Berufserfahrung liegt zwischen einem halben Jahr und 33 Jahren. Die Interviews kamen vorwiegend über Kontakte der Studierenden aus ihren Praxissemestern zustande. Es wurden Informationen zur Person (s. oben) erhoben und Leitfadeninterviews mit durchweg offenen Fragen verwendet. Beides bewerkstellig- 
ten Studierende, die dafür durch die Autorin geschult worden waren. Mit den von ihnen Interviewten hatten die Studierenden keine persönliche oder berufliche Bindung. Die Studierenden fertigten Tonaufzeichnungen von den Interviews an, transkribierten diese in Word-Dateien, sodass die so gewonnenen Texte von der Autorin nach einem inhaltsanalytischen Verfahren, das auch Quantifizierungen erlaubt (Mayring 2015), ausgewertet werden konnten.

Von den Interviewergebnissen wurden zunächst nur die Antworten auf die Kernfrage: „Was macht aus Ihrer Sicht professionelles Handeln als Sozialarbeiter*in aus?" betrachtet. Aufgrund dessen erfolgten Auswertungen der Antworten zu sechs weiteren in diesem $\mathrm{Zu}$ sammenhang relevanten Fragen: zu Exemplifizierungen von methodischem Vorgehen, zur Selbstreflexion, zur Fort- und Weiterbildung, zu Supervision und anderen qualitätsentwickelnden Maßnahmen.

\section{Professionelles Handeln}

Die verschiedenen Aspekte in den Antworten lassen sich in vier Themenblöcke gliedern. Alle von den Interviewteilnehmer_innen genannten unterscheidbaren Aspekte einer Antwort wurden einzeln gezählt. Ein Beispiel: „Ich finde es ganz wichtig, dass man eine gute Beziehung zu den Klienten aufbaut (M, Z. 211), dass man empathisch mit ihnen arbeitet (M, Z. 211-212), aber dennoch den Klienten klare Grenzen aufzeigt ( $\mathrm{M}$, Z. 212)“ werden als drei Aspekte verstanden. Wenn man die Themenblöcke nach ihrer Anzahl der ihnen zugeordneten Aspekte ordnet, ergibt sich folgende Rangfolge (Häufigkeiten in Klammern): Methodenwissen (42), Qualitätssicherung (31), Einhaltung der Professionsethik (12), theoretische Grundlagen (6).

Hier einige Beispiele, um zu illustrieren, welche Art von Antworten welchem Themenblock zugeordnet wurden:

- Methodenwissen: verschiedene Methoden anwenden (4 Nennungen), Arbeitsprinzipien wie beispielsweise Balance zwischen Nähe und Distanz gegenüber Klient* innen praktizieren (4), Empathie üben können (4),

- Qualitätsentwicklung: sich reflektieren (12), sich fortund weiterbilden (9), sich supervidieren lassen (4),

- Professionsethik: Autonomie fördern (3), KlientInnen wertschätzen (2), ethische Richtlinien befolgen (2) und

- Theoretische und wissenschaftliche Grundlagen: wissensbasiert handeln (3), nach Wichtigkeit unterscheiden (1), Handeln begründen können (1).

Auf diese vier Themenkreise werden im Nachfolgenden vertiefte Blicke geworfen. Es sei bereits darauf hin- gewiesen, dass mit diesen vier Themen all jene Elemente angesprochen sind, die im wissenschaftlichen Diskurs der Sozialen Arbeit als konstitutiv für „Professionalität“ angesehen werden (z. B. Dewe und Otto 2018; Borrmann 2016; von Spiegel 2018; Heiner 2004; Staub-Bernasconi 2017).

\section{Betrachtung von "Methodenwissen"}

Die Antwortkategorie „Methodenwissen“ hat die meisten Nennungen erfahren. Um hier Genaueres in Kenntnis zu bringen, wurden die Antworten auf eine weitere Interviewfrage betrachtet. Die Frage lautet: „Könnten Sie mir ein Beispiel dafür geben, wie Sie methodisch bei einem konkreten Fall genau vorgehen? “.

Alle Antworten verdeutlichen, dass sie über große Handlungs- und Entscheidungsspielräume verfügen und dies auch als sachlich notwendig beurteilen. Teilweise gibt es Vorgaben, wie gesetzliche Grundlagen, die das Handeln leiten und damit Standards vorgeben. Bei der Schilderung des konkreten Vorgehens zeigt sich, dass fast alle ein planvolles Vorgehen beschreiben und sie individuell auf die Problemlagen ihrer Klient_innen eingehen. Am Beispiel Jugendamt: Dessen Mitarbeiter_ innen beziehen sich immer auf ihr Vorgehen bei Verdacht auf Kindeswohlgefährdung. Ihr diesbezügliches Handeln scheint eingebettet in ein recht klares Konzept, das den diagnostischen Prozess regelt - bis hin zur endgültigen Klärung, ob Kindeswohlgefährdung vorliegt oder eben nicht. Zur Unterstützung gibt es klare Dienstanweisungen, Handreichungen des Landesjugendamtes (Prüfschema bei Kindeswohlgefährdung) sowie eine spezifische Software. Das bedeutet allerdings nicht, dass die Sozialarbeiter_innen sich eines Fachvokabulars wie etwa „Soziale Diagnostik“ bedienen oder von einem „Plan“ sprechen würden. Es heißt, dass sie ihren Beschreibungen nach zunächst zu verstehen suchen, worum es geht; um dann Ziele zu vereinbaren, die sie anschließend zum Beispiel mit Hilfe von „Ressourcenarbeit“ (P, Z. 312) gemeinsam mit den Klient_innen anstreben. Bei Nichterreichen der Ziele, beginnt der Prozess von Neuem. Ein unterstützender Beziehungsaufbau wird dabei für wesentlich erachtet.

\section{"Qualitätsentwicklung" unter der Lupe}

Für ihr professionelles Handeln ebenfalls sehr bedeutsam halten die Praktiker_innen neben Methodenwissen die "Qualitätsentwicklung“. Bei der Auswertung ließen sich der Qualitätsentwicklung vor allem zuordnen: (Selbst-)Reflexion, Fort- und Weiterbildung, Supervision und andere qualitätsentwickelnde Maßnahmen. Für einen vertieften Blick wurden die Antworten auf fünf weitere Fragen von thematischer Relevanz betrach- 
tet. Die Ergebnisse dieser Analyse sind nachfolgend dargestellt.

\section{(Selbst-)Reflexion}

Eine der Fragen lautete, für wie wichtig Selbstreflexion bzw. Selbsterfahrung. angesehen wird, wenn es um die eigene Professionalität geht. Drei Viertel der Befragten heben die Bedeutung von Reflexion hervor, und damit gemeint ist sowohl die Reflexion der eigenen Person wie die des eigenen Handelns gegenüber den Klient_innen. „Wie agiere ich, hab' ich mir das gut überlegt, kann ich begründen, warum ich wie agiere? Ja das ist ja oft so und dazu gehört natürlich, dass ich weiß, ich hab' jetzt so agiert, weil ich kann diesen Mann nicht leiden (lachen) zum Beispiel.“ (P, Z. 474-489).

Auffällig oft geht es darum zu reflektieren, wie man in einem Fall oder in einer schwierigen Situation weitermachen könnte. Das Know-How steht im Vordergrund. Elf Befragte finden Selbsterfahrung in Form von Weiterbildungsbausteinen, Teamsitzungen oder Supervisionen ganz wichtig, wenn es um professionelles Handeln geht; die anderen scheinen ihr keine besondere Bedeutung beizumessen. „Also ich denke, dass es sehr wichtig ist vor allem im sozialen Bereich, dass man sich mit sich selber auseinandersetzt und eben genau der Bereich Selbsterfahrung einen hohen Stellenwert hat. Weil je mehr man sich einfach selber kennt, und je mehr man seine eigenen Probleme, seine eigenen, ja, was da alles so schlummert in einem, genau. Desto professioneller kann ich auch einfach mit den Klienten umgehen. Ich denke schon, dass das sehr wichtig ist in der Sozialen Arbeit." (A, Z. 106-110).

\section{Fort- und Weiterbildung}

Zwei Interviewfragen thematisieren Fort- und Weiterbildung. Mehr als die Hälfte der Befragten begreifen diese als Qualitätsentwicklungsmaßnahme. Und das in dem Sinne, dass sie die berufliche Weiterqualifizierung als Möglichkeit zur Erhöhung ihrer methodischen Handlungskompetenz sehen. „Und da muss man einfach auch am Ball bleiben und ja professionell, das ist professionelles Handeln. Sich auch Handwerkszeug aneignen. Eben nicht nur aus dem Bauch raus agieren.“ (C, Z. 326-327).

Der Erfahrungshintergrund in Sachen Fort- und Weiterbildung lässt sich kurz so skizzieren: Bis auf zwei Berufsanfänger_innen bildeten sich alle weiter. Fünf Sozialarbeiter_innen verfügen über mindestens eine mehrjährige Weiterbildung (z. B. Traumatherapie, Systemische Familientherapie) und zusätzlich - so wie auch die anderen Interviewten - über mehrere Fortbildungen.

\section{Supervision}

Zur Supervision gab es eine Interviewfrage, die erstens erfasste, ob Supervision überhaupt in Anspruch genommen wird und zweitens eruierte, ob man sie als Hilfe empfindet.

Zwei der Befragten haben momentan keine Supervision und weitere zwei berichten, sie könnten Fall- oder Teamsupervision auf Anfrage erhalten. Zwei Drittel sind ca. alle vier bis sechs Wochen routinemäßig in Team-Supervision; einige können zusätzlich Einzelsupervision erhalten. Alle Interviewten finden Supervision sehr wichtig, um die Qualität ihrer Arbeit zu steigern. Als hilfreich erleben sie den Austausch mit Kolleg_innen, die Entlastung durch das Gespräch an sich sowie das Erfahren neuer Perspektiven, die von den Supervisor_innen eingebracht werden.

\section{Andere qualitätsentwickelnde Faktoren}

Eine weitere Interviewfrage bot die Möglichkeit, andere Faktoren zu benennen, die als förderlich für die Qualität angesehen werden. Dazu gehören etwa regelmäßige Teamsitzungen oder andere sonstige kollegiale Gespräche. Auf der Organisationsebene seien Instrumente wie Qualitätsmanagementhandbücher, Ordner mit selbst entwickelten Ablaufplänen (z. B. Erstellung einer Vermisstenanzeige) oder auch Dienstanweisungen (z. B. Umgang bei Verdacht auf Kindeswohlgefährdung) im Einsatz, die auch genutzt würden. Jede_r Studienteilnehmer_in kann mindestens einen dieser besonderen qualitätsentwickelnden Faktoren nennen.

\section{Theoretische und wissenschaftliche Grundlagen}

Zum Themenblock „Theoretische und wissenschaftliche Grundlagen “ finden sich nur sechs Nennungen, die von lediglich vier Befragten stammen. Zum Beispiel: „Professionelles Handeln heißt, dass ich weiß, was ich tue. Und das geht nur indem ich mich theoretisch natürlich auch, mit bestimmten Themen beschäftige. Also das, ich brauche theoretische Grundlagen“ (C, Z. 391-401). Zur Prüfung dieses doch erstaunlichen (s. unten) Ergebnisses wurde Folgendes gemacht. Mit der entsprechenden Word-Funktion wurden alle Interviewtexte in Gänze nach den Wörtern „Theorie“, „theoretisch“, „Wissenschaft“, „wissenschaftlich “ durchsucht. Für „Theorie“ gab es fünf Funde; zwei davon waren eindeutig negativ konnotiert. Einmal verwendet eine Befragte das Wort „wissenschaftlich“ im Zusammenhang mit häuslicher Gewalt. Eine weitere Suche nach dem Wort „Wissen“ ergab dagegen elf Treffer, davon sechs in Zusammenhang mit Weiterbildung - im Sinne von Wissen erwerben wollen, wobei unklar bleibt, welche Art von Wissen gemeint ist. 


\section{Professionsethik}

Zum Themenblock „Professionsethik“ gab es über die Zentralfrage hinaus keine weitere Interviewfrage, die diesen Themenkreis näher beleuchtet hätte. Es bleibt also bei den für die Zentralfrage gefundenen Nennungen: Insgesamt 12 aus 9 Interviews. Aus der Perspektive der befragten Sozialarbeiter_innen bedeutet professionelles Handeln vor allem zweierlei: methodisch gut sein und die Arbeitsqualität fortlaufend erhöhen.

\section{Methodenwissen und Qualitätsentwicklung}

Inwiefern die Befragten über gediegene Kenntnisse zum Methodenwissen verfügen und wie sie ihr Wissen tatsächlich umsetzen (besser oder schlechter), kann mit dem vorliegenden Untersuchungsdesign nicht beantwortet werden. Es lässt sich jedoch schlussfolgern, dass sie professionelles Handeln zu einem großen Teil über Methodenwissen definieren. Die hohe Bedeutung, die der Handlungskompetenz allgemein von Sozialarbeiter innen beigemessen wird, lässt sich zusammengefasst bei Ohling (2015) nachlesen.

Die Steigerung bzw. Erhaltung der Arbeitsqualität wird meist gewährleistet durch Fort- und Weiterbildungen, Supervisionen, Teamgespräche und andere qualitätsentwickelnde Faktoren. Diese Faktoren zusammen sichern und steigern reflexive Kompetenz. Dewe und Otto (2018) sehen diese als zentral für Handeln in der Sozialen Arbeit an; das gilt heute als allgemein anerkannt.

\section{Theoretische und wissenschaftliche Grundlagen}

Anders verhält es sich bei den zwei anderen Themenblöcken. Auf die Frage nach den Vorstellungen von professionellem Handeln beziehen sich nur relativ wenige Aussagen der Interviewpartner_innen auf theoretische und wissenschaftliche Grundlagen sowie auf Professionsethik. Und auch bei der näheren Betrachtung von Methodenwissen und Qualitätsentwicklung fällt auf, dass es hier eher um Selbstreflexion geht und um neue bzw. andere Herangehensweisen als um den Einbezug von Theorie und Wissenschaft in die Überlegungen.

Dieses Doppelergebnis ist in den Kontext weiterer einschlägiger Studienergebnisse, die doch sehr selten sind, zu setzen. Beginnen wir mit dem Punkt „Theoretische und wissenschaftliche Grundlagen“.

Klüsche (1990) hat bereits vor 30 Jahren bei einer Befragung von 301 Sozialarbeiter_innen festgestellt, dass sich rückblickend die Hälfte in Sachen Theorie nicht ausreichend auf ihre berufspraktische Tätigkeit vorbereitet fühlt und sie ihr Handeln wenig an wissenschaftlichem Erklärungs- und Begründungswissen ausrichten. Es könnte sein, dass sich diesbezüglich noch nicht so viel verändert hat. Das rührt möglicherweise daher, dass sich Sozialarbeiter_innen auch schon im Studium nicht so sehr für theoretische und wissenschaftliche Grundlagen interessieren bzw. dafür von den Lehrenden interessiert werden (Bek 2020). Hierfür spricht ein Ergebnis des 12. Studierendensurveys: in der Fachgruppe Sozialwissenschaften an Fachhochschulen halten (Stand Wintersemester 2012/2013) lediglich neun Prozent Wissenschaft und Forschung für wichtig bzw. sehr wichtig (Ramm et al. 2014, S. 117, Tab. 13). Unterkofler (2018, S. 12) resümiert nach Betrachtung verschiedener Studienergebnisse, dass Sozialarbeiter_innen sich in der Praxis mehr an ihren Erfahrungen als an wissenschaftlichem Wissen orientieren.

Angesichts dessen überraschen zwei Ergebnisse einer 2018 durchgeführten Online-Befragung von überwiegend Sozialarbeiter_innen (Como-Zipfel et al. 2019) doch sehr. Dem Statement „Die Methoden und Arbeitsweisen der Sozialen Arbeit sollten wissenschaftlich begründet und erprobt sein" stimmen zwei Drittel (voll und ganz) zu ( $n=1306$; S. 28, Tab. 1$)$, bei „Die Wirksamkeit der in der Sozialen Arbeit eingesetzten Methoden und Arbeitsweisen sollte empirisch belegt sein (voll und ganz) etwas mehr als die Hälfte“ ( $n=1299$; ebd.). In beiden Fällen kommt eine hohe Wertschätzung für Wissenschaftlichkeit zum Ausdruck.

Es ist Aufgabe künftiger Forschung, der Unterschiedlichkeit der vorliegenden empirischen Befunde nachzugehen. Im Studium (Fachbereichstag 2016), in der Deutschen Gesellschaft für Soziale Arbeit (2016) und im Diskurs der Sozialen Arbeit (Engelke et al. 2018; Borrmann 2016) werden theoretische und wissenschaftliche Grundlagen für sehr wichtig erachtet, wenn es um professionelles Handeln geht.

\section{Professionsethik}

Abschließend zur Professionsethik: Von 16 Befragten haben insgesamt nur neun Aspekte von Professionsethik auf die Zentralfrage angesprochen. Die Zentralfrage lautete: „Was macht aus Ihrer Sicht professionelles Handeln als Sozialarbeiter* in aus? " Auch hier ist es Aufgabe künftiger Forschung, die offensichtliche Diskrepanz vorliegender empirischer Befunde zu klären. Die Notwendigkeit einer solchen empirisch fundierten Klärung ergibt sich aus der Bedeutung, die ihr im Studium (Fachbereichstag 2016), dem Deutscher Berufsverband für Soziale Arbeit (DBSH 2014) und im Diskurs der Sozialen Arbeit (Staub-Bernasconi 2017) beigemessen wird.

\section{Fazit}

Die Ergebnisse zeigen, dass die Praktiker_innen mehrheitlich auf reflektiertes Handeln sehr viel Wert legen 
und an der Erhöhung ihrer Arbeitsqualität interessiert sind. Die Interviews zeigen auch, dass sie planvoll vorgehen und dabei versuchen, individuell passende Lösungen mit und für die Klient_innen zu finden. Sie scheinen darüber hinaus über ausreichend Handlungsfreiheit zu verfügen. Es gelingt ihnen allerdings nicht, ihr Vorgehen professionell darzustellen, was einem Studienergebnis von Hansjürgens (2016) entspricht. Demnach haben Sozialarbeiter_innen Schwierigkeiten, Fachliches darzustellen. Sie verwenden stattdessen viele alltagssprachliche Umschreibungen. Mehr ins Gewicht fällt wohl, dass die Befragten nur einige wenige explizit theoretische, wissenschaftsbasierte und relativ wenige professionsethische Bezüge herstellen, wenn sie über ihr Handeln sprechen. Die Pflicht zur Begründung zählt aber zu den wesentlichen Merkmalen professionalisierter Praxis. Um in einen Fall professionell (auch methodisch professionell) tätig zu werden, benötigt eine Sozialarbeiter_in wissenschaftlich gewonnenes Erklärungs- und Begründungswissen bzw. Theoriewissen. Um zu verstehen, worum es geht und Klient_innen Alternativerklärungen anbieten zu können, die neue Handlungsmöglichkeiten für alle Beteiligten eröffnen. Möglicherweise ist auch dieses Wissen implizit vorhanden und wird in der Praxis sogar genutzt, kommt eben nur nicht in den Sinn, wenn nach professionellem Handeln gefragt wird oder es kann nicht fachlich angemessen zum Ausdruck gebracht werden.

Eingegangen. 5. Juni 2020

Angenommen. 7. August 2020

Funding. Open Access funding enabled and organized by Projekt DEAL.

Open Access. Dieser Artikel wird unter der Creative Commons Namensnennung 4.0 International Lizenz veröffentlicht, welche die Nutzung, Vervielfältigung, Bearbeitung, Verbreitung und Wiedergabe in jeglichem Medium und Format erlaubt, sofern Sie den/die ursprünglichen Autor(en) und die Quelle ordnungsgemäß nennen, einen Link zur Creative Commons Lizenz beifügen und angeben, ob Änderungen vorgenommen wurden.

Die in diesem Artikel enthaltenen Bilder und sonstiges Drittmaterial unterliegen ebenfalls der genannten Creative Commons Lizenz, sofern sich aus der Abbildungslegende nichts anderes ergibt. Sofern das betreffende Material nicht unter der genannten Creative Commons Lizenz steht und die betreffende Handlung nicht nach gesetzlichen Vorschriften erlaubt ist, ist für die oben aufgeführten Weiterverwendungen des Materials die Einwilligung des jeweiligen Rechteinhabers einzuholen.

Weitere Details zur Lizenz entnehmen Sie bitte der Lizenzinformation auf http://creativecommons.org/licenses/by/4.0/deed.de

\section{Literatur}

Bek, T. (2020). Wie Theorien in der Lehre schmackhaft machen? Über einen Crash-Kurs zu Beginn des Studiums Sozialer Arbeit. Soziale Praxis, 69(6), 210-216.

Borrmann, S. (2016). Theoretische Grundlagen Sozialer Arbeit. Ein Lehrbuch. Weinheim, Basel: Beltz Juventa.

Como-Zipfel, F., Kohlfürst, I., \& Kulke, D. (2019). Welche Bedeutung hat Ethik für die Soziale Arbeit? Berlin: Lambertus Verlag.

DBSH - Deutscher Berufsverband für Soziale Arbeit e. V. (2014). Berufsethik des DBSH. Ethik und Werte. Forum Sozial 4, S. 1-43. https://www. dbsh.de/media/dbsh-www/redaktionell/pdf/Sozialpolitik/DBSH-Berufsethik-2015-02-08.pdf. Zugegriffen: 8. Juni 2020.

Deutsche Gesellschaft für Soziale Arbeit (2016). Kerncurriculum Soziale Arbeit. Eine Positionierung der Deutschen Gesellschaft für Soziale Arbeit. https://www.dgsa.de/fileadmin/Dokumente/Aktuelles/DGSA_Kerncurriculum_final.pdf. Zugegriffen: 7. Aug. 2020.

Dewe, B., \& Otto, H.-U. (2018). Professionalität. In H.-U. Otto \& H. Thiersch (Hrsg.), Handbuch Soziale Arbeit (S. 1203-1213). München: Ernst Reinhardt Verlag.

Ehlert, G. (2019). Professionalisierung. socialnet Lexikon. Bonn. Socialnet. https://www.socialnet.de/lexikon/Professionalisierung. Zugegriffen: 7. Aug. 2020.

Engelke, E., Borrmann, S., \& Spatscheck, C. (2018). Theorien der Sozialen Arbeit. Eine Einfübrung (7. Aufl.). Freiburg i. Breisgau: Lambertus.

Fachbereichstag Soziale Arbeit (2016). Qualifikationsrahmen Soziale Arbeit Version 6.0. https://bagprax.sw.eahjena.de/data/stellungnahmen/weitere/QR_SozArb_Version_6.0.pdf. Zugegriffen: 7. Aug. 2020.

Hansjürgens, R. (2016). Soziale Arbeit in der ambulanten Suchthilfe. Optionen zur Professionalisierung und fachlichen Inszenierung als gleichwertige Partnerin in einem multiprofessionellen Feld. Coburg: ZKS-Verlag Internet.

Heiner, M. (2004). Professionalität in der Sozialen Arbeit. Theoretische Konzepte, Modelle und empirische Perspektiven. Stuttgart: Kohlhammer.

Klüsche, W. (1990). Professionelle Helfer. Anforderungen und Selbstdeutungen. Aachen: Kersting.

Mayring, P. (2015). Qualitative Inhaltsanalyse: Grundlagen und Techniken (12. Aufl.). Basel: Beltz.

Ohling, M. (2015). Soziale Arbeit und Psychotherapie. Veränderung der beruflichen Identität von SozialpädagogInnen durch Weiterbildungen in psychotherapeutisch orientierten Verfahren. Weinheim: Beltz Juventa.

Pantucek-Eisenbacher, P. (2015). Bedrohte Professionalität? In S. Busse, G. Ehlert \& S. Müller-Hermann (Hrsg.), Bedrohte Professionalität. Einschränkungen und aktuelle Herausforderungen für die Soziale Arbeit (S. 29-42). Wiesbaden: Springer VS.

Ramm, M., et al. (2014). Studiensituation und studentische Orientierungen. 12. Studierendensurvey an Universitäten und Fachhochschulen. Konstanzer Online-Publikations-System (KOPS). http://nbn-resolving.de/urn:nbn :de:bsz:352-0-265900. Zugegriffen: 7. Aug. 2020.

Scherr, A. (2018). Professionalität - ein Qualitätsmerkmal von Organisationen. Warum es nicht genügt, Fachkräfte als Träger professioneller Kompetenzen zu adressieren. Sozial Extra, 42(1), 8-13. https://doi.org/10.1007/ s12054-017-0108-6.

Staub-Bernasconi, S. (2017). Soziale Arbeit als Handlungswissenschaft. Systemtheoretische Grundlagen und professionelle Praxis (2. Aufl.). Bern, Stuttgart, Wien: Haupt.

Unterkofler, U. (2018). Professionsforschung in der Sozialen Arbeit. In C. Schnell \& M. Pfadenhauer (Hrsg.), Handbuch Professionssoziologie (S. 1-21). Wiesbaden: Springer. https://doi.org/10.1007/978-3-658-131548_20-1.

Von Spiegel, H. (2018). Methodisches Handeln in der Sozialen Arbeit. Stuttgart: UTB. 